\title{
Application of Reverse Engineering in Design of 81/2" Torsional Impact Bit
}

\author{
Li Xiaojun ${ }^{1,2}$, Wang Deguo ${ }^{1}$, Zhang Junfeng ${ }^{2}$, Zhao Jibin $^{2}$, Wang Feiyue ${ }^{2}$ \\ ${ }^{1}$ Mechanical and Electrical Engineering, China University of Petroleum, Beijing 1022493, China \\ ${ }^{2}$ Western Drilling Engineering Technology Research Institute, Karamay, Xinjiang 834000, China
}

Keywords: Hard Strata; Reverse Design; Torsional Impact Bit; Bit Tooth Arrangement

\begin{abstract}
To solve the problem of drilling difficulty of hard strata in China's Yumen Oilfield and so on, torsional impact drilling tools are widely used in the field. This paper mainly describes the reverse design process of $812^{\prime \prime}$ torsional impact bit, to coordinate the torsional impact drilling. Through establishing bit CAD parametric model, analyze the model deviation and curvature, to rationally adjust the design parameters, so that obtain the bit entity model. At last, we recommend applying Pro/ENGINEER for local adjustment of bit tooth arrangement, and describe the main features of the drill bit.
\end{abstract}

\section{Introduction}

China's Yumen Oilfield as an old oil field which has been developed for 70 years is very urgent for drilling speeding up, especially for Qingxi Oilfield. The main difficulty in Qingxi Oilfield speeding up shows in the Cretaceous strata, and with $30 \%$ of footage consumption and $60 \%$ of drilling cycle, it has a great potential to be tapped. The difficulties mainly reflect in the following aspects: (1) the rock is hard and fragile, so it is difficult to drill into the stratum; (2) fracture developing, fault converging, the drilling fluid soaks the stratum for long time, and there are many complex accidents; (3) rich in pyrite and gravel, which will wear the drilling bit. In the process of PDC cutting the stratum Cretaceous wells 4200 meters below, by restriction of processes and tools, the composite sheet happens brittle chipping, leading to rapid damage. Faced with the drilling problems of Yumen Oilfield, we carry out study on reverse remodeling of $81 / 2$ " torsional impact bit by world's leading software Rapidform XOR, and finally design the rigid drill bit to coordinate the use of torque impactor, so that greatly improve the drilling speed, and extend the single net drilling time, and meanwhile the drill bit of such structure has good reparability ${ }^{[1,2,3]}$.

The software used in our design includes Geomagic Design X and Pro/ENGINEER. Geomagic Design $\mathrm{X}$ is used to complete parametric solid model of 3d drill bit point cloud model by scanning. The model has editability, because it is able to transform the entity model into Parasolid、STEP 、 IGES and STL, which can be used in any CAD/CAM/CAE application. When the point cloud model obtained through scanning constructs a complete solid model in Geomagic Design X, it usually spends less time than direct design in CAD.

\section{PDC Drill Bit Point Cloud Data Processing}

Import of PDC Drill Bit Point Cloud Data and Streamline Point Cloud Data. Geomagic Design X uses solid and surface modeling techniques, from CAD model construction of PDC drill bit to polygon mesh, the obtained static NURBS surface can be edited 

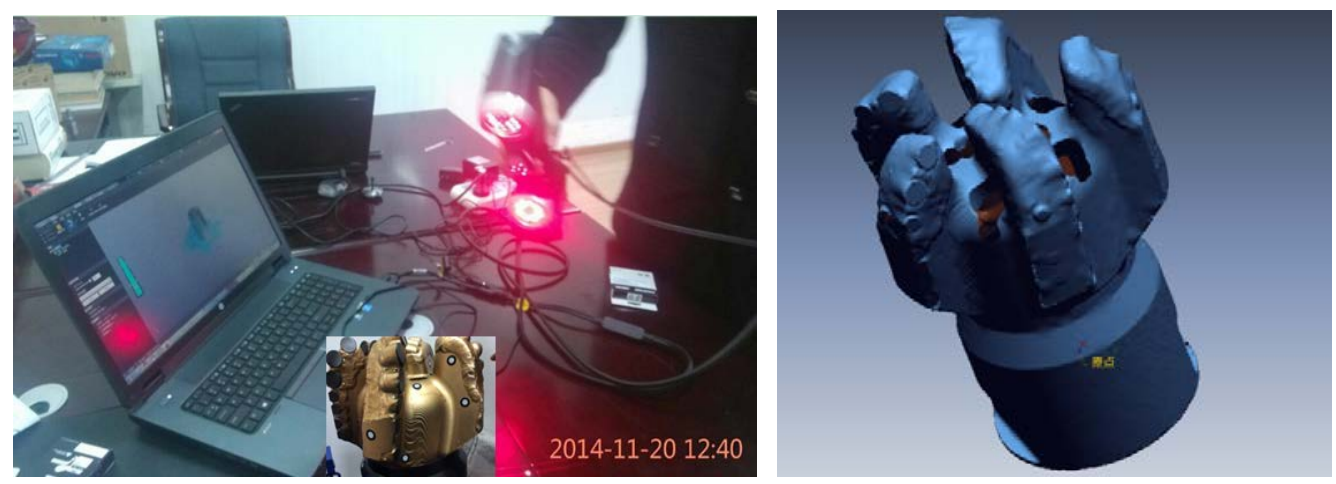

Figure 1. Engineers are scanning the PDC drill bit

Field Division and Patch Fitting. The purpose drill bit point cloud field division is to obtain independent patch fitting area, which can divide the point cloud into 5 blades and body drill bit, and the purpose of local refinement of 5 blades tooth position is to obtain the specific location of the teeth. Field division is very important for patch fitting. It needs to apply filter to edit and strengthen the point cloud of sweep surface, and carry out delete, sampling and smoothing operation on the miscellaneous store, and then conduct DEM analysis, machining, RP on the obtained patch.
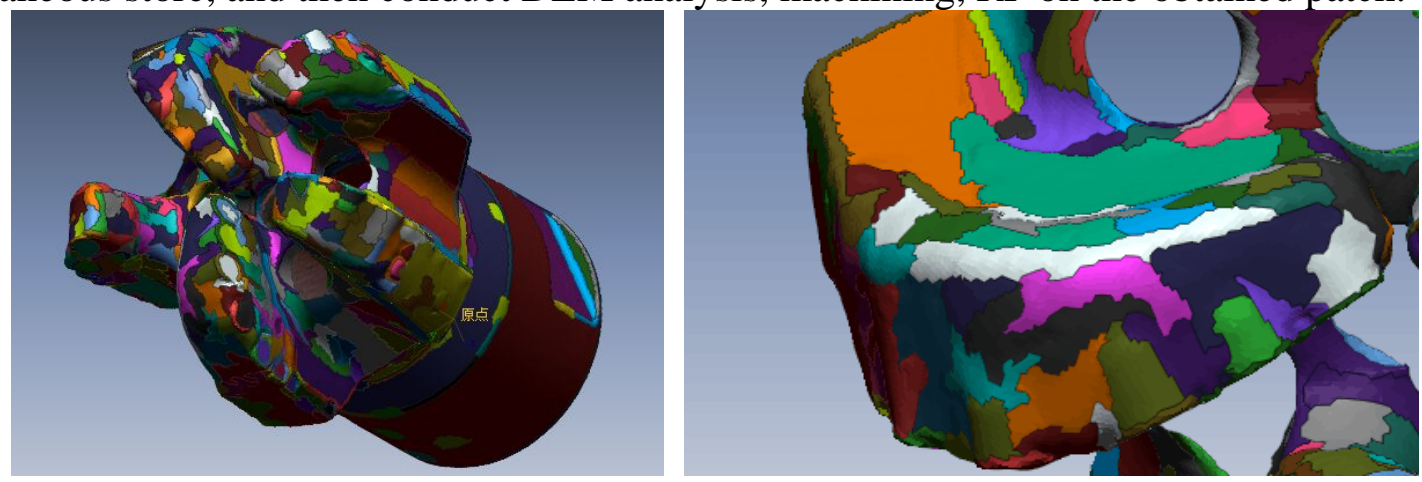

Figure 2. Field division and local graphic of torsional impact bit

Fitting of Characteristic Curve, Boundary Curve and Curved Surface. Based on the existing point cloud, extract key section of the point cloud, and fit it into curve, and then carry out three-dimensional stretching, rotating, draft, split and other operations on the curve to generates three-dimensional body. As for curved surface, we can directly adopt the way of patch fitting to generate curved surface, and finally materialize the curved surface.

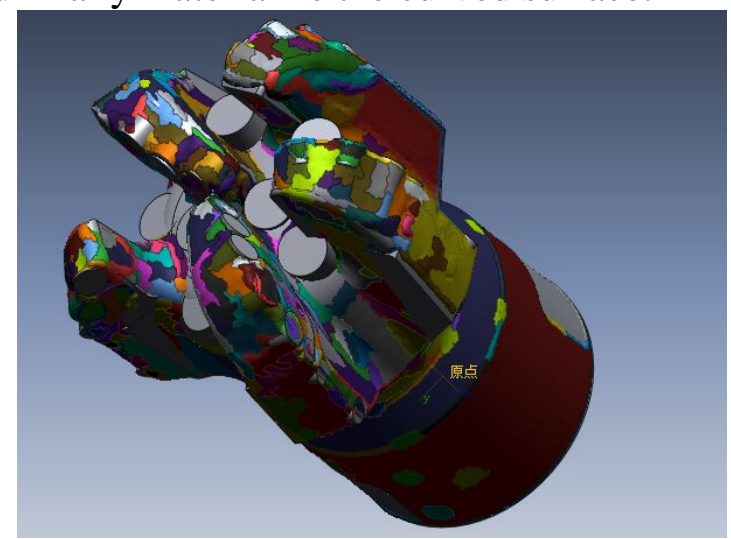

Figure 3. Model reconstruction by way of curved surface fitting

Model Error Analysis. The purpose of model image error comparison is to analyze the error between the model and the original 3D scanning data obtained by Geomagic Design X, including the size of the curvature. The error in our design is basically between -0.8 and 0 , and change of curvature is basically between 0 and 0.2 . For the part where the curvature doesn't meet the design requirements, we can we can modify the design parameters of the existing parts. The main reason 
for curvature examination is there may be many small planes while curved surface fitting, which may cause late programming disorder and then improve the processing cost, so we need to carefully analyze the small parts, and on the basis of meeting the drill function realization, we should simplify the structure as far as possible.
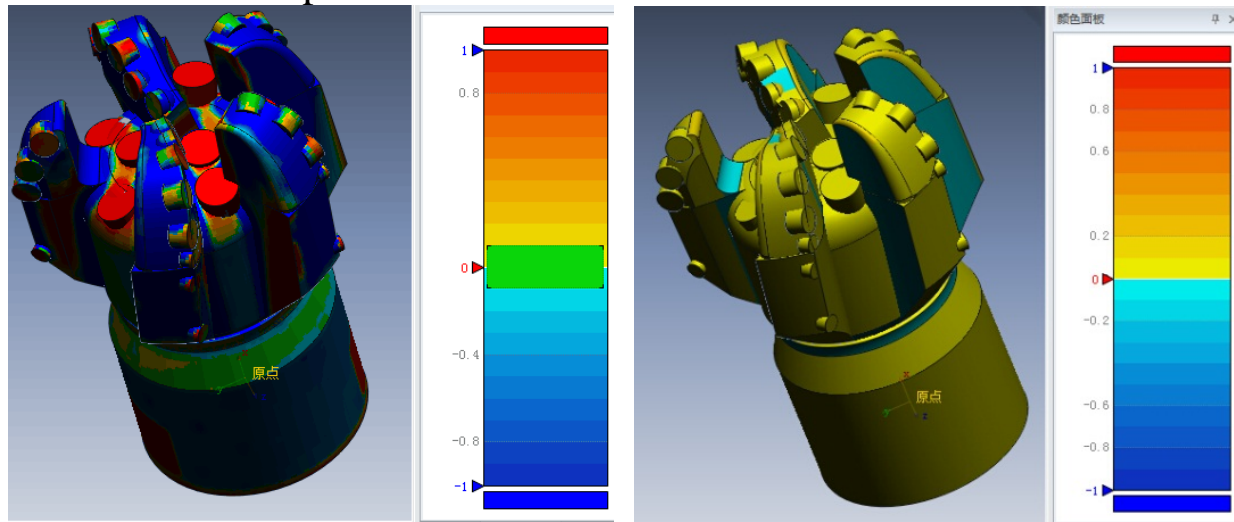

Figure 4. Error analysis and curvature examination in design of PDC bit

\section{Principles of Cutting Tooth Adjustment}

Apply the command LiveTransfer(TM) to import the obtained drill bit 3D model into software Pro/ENGINEER, and complete local optimization of the tooth. There are some principles of cutting tooth adjustment as follow:

(1) Rake angle of cutting tooth

Rake angle of cutting tooth of PDC drill bit plays an important role in improving cutting efficiency, protecting cutting tooth, and prolonging life, to improve the drilling speed. It is widely believed that, tooth rake angle reduction can effectively help for cutting. On the contrary, if the tooth rake angle increases, then cutting tooth will be durable in hard stratum and the drill life is long, but mechanical drilling speed and cutting efficiency will decrease. For the PDC drill bit coordinated with torque impactor, the tooth rake angle should be large enough, so as to prevent the damage caused by the impact of hard stratum.

(2) Side angle of cutting tooth

Side angle of cutting tooth of PDC drill bit conducive to cleaning of the debris and transfer of the drill bit. Once been sheared, the debris will take the initiative to transfer to the chip groove and the annular space. Side angle has good effect on solving the mud problems of adhesive shale and shale formation. The side angle should be variable, and in design we can adjust the side angle to promote drill bit cleaning and then improve the drilling efficiency ${ }^{[4,5,6]}$.

GENERAL characteristics OF $81 / 2^{\prime \prime}$ TORSIONAL IMPACT BIT

\begin{tabular}{|c|c|c|c|c|c|c|c|}
\hline \multirow{2}{*}{$\begin{array}{c}\text { Serial } \\
\text { number }\end{array}$} & \multirow{2}{*}{ Size } & \multirow{2}{*}{ Model } & \multirow{2}{*}{$\begin{array}{c}\text { Tooth } \\
\text { number }\end{array}$} & \multirow{2}{*}{ Ball head } & \multicolumn{2}{|c|}{ Cylinder } & $\begin{array}{c}\text { Gage } \\
\text { front }\end{array}$ \\
\cline { 5 - 8 } & & & & 1616 & 1613 & 1308 & 1308 \\
\hline 1 & $8-1 / 2$ & 516 & 55 & 4 & 22 & 9 & 20 \\
\hline
\end{tabular}

Table 1. Tooth arrangement of each blade

\begin{tabular}{|c|c|c|c|c|c|c|c|c|c|c|}
\hline Blade number & 1 & 2 & 3 & 4 & 5 & 6 & 7 & 8 & 9 & $\begin{array}{c}\text { Gage front } \\
1308\end{array}$ \\
\hline First blade & 1616 & 1616 & 1613 & 1613 & 1613 & 1613 & 1613 & 1308 & & 4 \\
\hline Second blade & 1613 & 1613 & 1613 & 1613 & 1308 & 1308 & & & & 4 \\
\hline Third blade & 1613 & 1613 & 1613 & 1613 & 1308 & 1308 & & & & 4 \\
\hline Forth blade & 1616 & 1616 & 1613 & 1613 & 1613 & 1613 & 1613 & 1308 & 1308 & 4 \\
\hline Fifth blade & 1613 & 1613 & 1613 & 1613 & 1308 & 1308 & & & & 4 \\
\hline
\end{tabular}

Formula of the number $\mathrm{i}+1$ tooth center abscissa (axial radius) is shown as follow: 


$$
R_{c(i+1)}=\sqrt{C+\left(R_{c(i)}-\frac{\theta_{2}}{360} \times \sigma\right)^{2}}-\frac{\theta_{1}}{360} \times \sigma
$$

Formula of cutting teeth axial height of class B dual-cone crown is shown as follow: Inner cone section:

$$
H_{c(i)}=k_{1} \times R_{c(i)}+b_{1}+\left(r_{c(i)}-l c_{i}\right) \sqrt{\left(1+k_{1}^{2}\right)}
$$

Crown arc section:

$$
H_{c(i)}=r-\sqrt{\left(r+l c_{i}-r_{c i}\right)^{2}-\left(R_{c(i)}-r_{0}\right)^{2}}
$$

Outer cone section:

$$
H_{c(i)}=k_{2} \times R_{c(i)}+b_{2}+\left(r_{c(i)}-l c_{i}\right) \sqrt{\left(1+k_{2}^{2}\right)}
$$

The differences between axial radiuses of all the gauge teeth are not obvious, so we unity it as:

$$
R_{c(i)}=d+d m l_{i}-r_{c(i)}
$$

In the formula, $d m l_{i}$ is the grinding amount of number $i$ gauge teeth.

Finally we determine the tooth arrangement mode as follow:
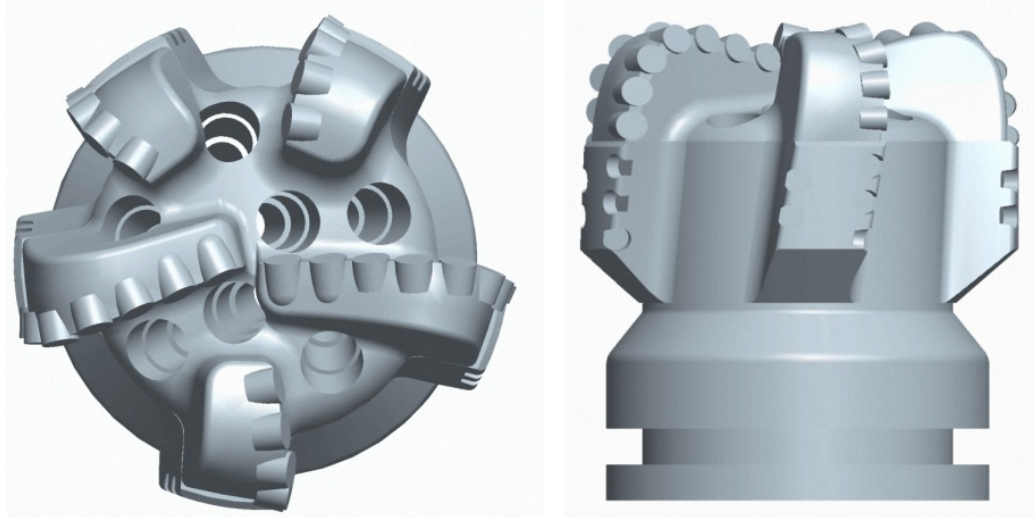

Figure 5. Tooth arrangement mode

The improved $81 / 2 "$ torsional impact bit has the characteristics as follow:

(1) The adjusted main cutting tooth rake angle is relatively large, which adapts to the middle stratum of thin interlayer with high compressive strength and certain grinding performance, such as marl, shale, anhydrite, calcareous sandstone and so on;

(2) Has high attack performance in soft or medium stratum, with the use of torsion impactor, reduce the wear of teeth $\mathrm{PDC}$, and adapts to abrasive for stratum with high compressive strength;

(3) The seven nozzle diameter can be adjusted according to need;

(4) The gauge structure can be adjusted.

\section{Conclusion}

Apply software Geomagic Design X for reverse design of the drill bit coordinating with torsion impactor, and carry out parameter inverse, can improve the design and manufacture speed of PDC drill bit, and achieve personalized changes on the reverse products, and finally obtain the drilling tool which can be used in special stratum, with good impact resistance and wear resistance.

\section{REFERENCE:}

[1] Rolf Pessier and, SPE, Baker Hughes; Michael Damschen, P.E., SPE, Baker Hughes. Hybrid Bits Offer Distinct Advantages in Selected Roller Cone and PDC Bit Applications [R]. IADC/SPE 128741-MS, 2010. 
[2] J.Clegg. Faster, Longer, and More-Reliable Bit Runs With New-Generation[R]. IADC/SPE 102067-MS, 2006.

[3] Yoosuf S, Goebel T.Right Bit, Right Application, Right Time: Case Studies of Enhanced Bit Design Process[R].SPE 106942, 2007.

[4] Bejarano C A, Munoz G, Perez G, et al. Case history-application of a new PDC bit design in deep Cretaceous and Jurassic hard formations in southern Mexico[R]. SPE 102232,2006.

[5] Sun Mingguang,Zhang Yunlian,Ma Dekun.Design and application of the PDC bit suited for multi-interbedded formation[J].Acta Petrolei Sinica,2001,22 (5):95 99.

[6] Gao Shaozhi, Zhang Jianhua, Li Tianming, et al.Research on PDC bit for drilling in gravel interlayer [J]. Oil Drilling \& Production Technology,2006,28 (4):20 21+28. 